\title{
An Experimental Study of the Effect of a Baffle on the Flow Structure in a Rectangular Open Channel Using UVP*
}

\author{
Hamidreza JAMSHIDNIA ${ }^{* * 1}$ and Yasushi TAKEDA** \\ ** Laboratory of Flow Field System Engineering, Division of Energy and Environmental Systems, \\ Graduate School of Engineering, Hokkaido University, N13W8, Sapporo, 060-8628, Japan \\ ('Corresponding author, E-mail: hamid@ring-me.eng.hokudai.ac.jp)
}

\begin{abstract}
A flow structure around an intermediate standing baffle in a rectangular open channel has been investigated experimentally. The instantaneous vertical velocity components were successfully measured using an Ultrasonic Velocity Profiler (UVP). Various spatial distributions such as profiles of the vertical time-averaged velocity and relative turbulent intensities at various vertical measuring lines around the baffle indicate how the flow structure changes from up- to downstream of the baffle. At the baffle's upstream they indicate the flow structure of the uprising flow. But behind the baffle indications of vortex shedding and flow separation such as the prominent peak values in the relative turbulent intensity profiles is observed. Also, spatio-temporal distributions of the vertical velocity at up- and downstream sections confirm the existence of periodic change of flow direction near the edge of the baffle at its downstream which can be attributed to the vortex shedding from the baffle edge. In addition, space-dependent power spectra indicate the existence of some peak structures near the baffle edge height at its downstream. For these sections existence of peak values in the space distribution of two frequency modes could be confirmed corresponding to the vortex shedding due to the existence of the baffle. Furthermore, by using multi-line method and multiplexer the peak of the absolute value of the normalized two-point cross-correlation coefficients between vertical velocity fluctuations could be obtained to evaluate the effect of the baffle on the degree of correlation between vertical velocity fluctuations at upstream points with that of downstream ones. It has been found quantitatively that a baffle acts as a barrier wall and causes the degree of correlations to be decreased significantly from the vicinity of its edge height to the channel bed. Also existence of a local peak region in between the baffle edge height and free surface was found in color maps of degree of correlation. It was found that the degree of correlation decreases gradually from the peak region to the proximity of the baffle edge height. Also the decrease in the degree of correlation from that peak to the free surface was captured which can be attributed to the effect of free surface. Thus, flow around the baffle can be characterized into two regions with very different characteristics.
\end{abstract}

Key words: Flow Measurement, Velocimetry, Flow Pattern, Turbulent Flow, Separation, Baffle, UVP

\section{Introduction}

Understanding of flows over obstacles and steps is of great value due to 
their relevance to many practical and theoretical problems. For example, separated flows produced by an abrupt change in geometry are of great importance in many engineering applications ${ }^{(1)}$ such as power generating equipment, diffuser, electronic and turbine blade cooling, atmospheric flows over fences and hills and sudden area change in pipes and ducts ${ }^{(2)}$.

Flow over a Backward-Facing Step (BFS) has been the subject of numerous investigations due to its wide range of applications in engineering such as cooling techniques or heat transfer of electronic devices ${ }^{(3)}$. As an important example spatial-temporal velocity fields of the flow through an axisymmetric sudden expansion were experimentally investigated over a range of Reynolds numbers ${ }^{(4,5,6,7,8)}$. Also various researchers have studied flow field characteristics and its topology over a Forward-Facing Step (FFS) ${ }^{(9,10,11,12)}$. Specifically horizontal channel flows over FFS and BFS have been the focus of various studies ${ }^{(13,14)}$.

The flow over a standing baffle (step) can be regarded as an extreme combination of the flows over BFS and FFS. Importantly streamlines are not parallel to the wall at the separation point. Thus the impinging flow at immediate upstream of the baffle has two dimensional characteristics.

Although the geometry of a baffle is very simple, the flow over it has diversified time-dependent features and a complex flow structure, which in turn makes its investigation challenging and interesting.

From another perspective, provision of baffles or deflectors in settling tanks to modify the flow field and consequently to improve the efficiency of such tanks has been investigated by several researchers as a method for flow control. Since performance of a settling tank depends on its flow field, investigating the flow field structure is of great value ${ }^{(15)}$. Their performance is influenced by the turbulence level and hydraulic phenomena such as recirculation ${ }^{(16,17)}$. Lyn and Rodi ${ }^{(18)}$ conducted turbulence measurements by a Laser Doppler Anemometry (LDA) system in a rectangular laboratory settling tank and studied the flow fields from a plane jet impinging on two types of deflector at constant depth and discharge to obtain a better understanding of the hydrodynamics. Studies of Bretscher et al. ${ }^{(19)}$ on velocity and concentration fields in a rectangular settling tank with a central barrier wall (Intermediate baffle) showed the effectiveness of the baffle.

Ahmed et al. ${ }^{(16)}$ have investigated the effect of positioning and height of baffles on the flow pattern and suspended solid distribution qualitatively in a sedimentation laboratory tank by inserting the baffle from the top at three different positions from the inlet weir and with various heights. They studied both clean and suspension waters. It was found that the baffle position has a significant effect on the flow patterns and suspended solid concentration by influencing the direction of flow and affecting the size of dead zones. They also have found an optimum case for the baffle position and contraction ${ }^{(16)}$.

Taebi-Harandy and Schroeder ${ }^{(20,21)}$ found that effectiveness of baffle on the clarifier performance is dependent strongly on the predominant flow pattern. Tamayol et al. ${ }^{(22)}$ studied the effect of baffle configuration on the hydraulic performance of the primary settling tanks and determined the optimal position of the baffle. Firoozabadi et al. ${ }^{(23)}$ investigated the effect of the baffle and its position on the spatial structure of flow in a rectangular primary settling open channel, experimentally. They showed the possibility of the improvement of the flow field in the case of neutral flow for some 
baffle positions. Jamshidnia ${ }^{(24)}$ has studied the flow pattern in a rectangular sedimentation tank by an Acoustic Doppler Velocimeter (ADV) and investigated the possibility of modifying the flow field by a baffle for a better sedimentation. Jamshidnia et al. ${ }^{(25)}$ have investigated the effect of a standing baffle on the spatial and temporal structure of the flow along a laboratory rectangular open channel by an ADV. They also showed the existence of a highly spatial flow pattern at downstream portion of the baffle.

Jamshidnia and Takeda ${ }^{(26)}$ investigated the flow structure around a baffle by scanning a single UVP transducer along the free surface of a rectangular open channel at various streamwise positions and could successfully capture the various flow phenomena. Also their results show existence of a notable two-dimensionality and spatial variation in flow structure around the baffle. In their investigations the transducer was oriented $10^{\circ}$ inclined from the vertical direction. Therefore, it was not possible to decompose the flow components to extract vertical and/or streamwise velocities because only the component of velocity vector on the propagation direction of ultrasound beam could be obtained. Thus, to study this two-dimensional flow and to provide a more detailed quantitative picture of spatial variation in flow structure (uniform flows do not vary spatially, whereas non-uniform flows $\mathrm{do}^{(27)}$ ) and also to investigate the baffle effect on the flow structure quantitatively, we have found it important and necessary to extract the vertical velocity components. At the same time, one way to look at the degree of two-dimensionality in the flow around a baffle and its quantification is by investigating the vertical structure of the flow. This is especially the case when we investigate the flow structure in view of sedimentation process.

Also, to further confirm if the previous results are in agreement with the results corresponding to vertical structure of flow the components of velocity in the vertical measuring lines are obtained by UVP in the present study.

Furthermore, it should be noted that, investigation of the degree of correlation between fluctuations of vertical velocities at upstream points of the baffle with that of downstream points is important to achieve a better detailed understanding of the effect of the baffle and the flow behavior around it in a quantitative manner. For such correlation analysis the velocity components on the vertical measuring lines should be available. Thus it is essential to conduct the measurements for extracting the velocity component in vertical measuring lines. To the authors' knowledge, in this paper, we are going to present the first measurements of such correlation coefficients for flow around a standing baffle in a rectangular open channel. It is noted that the results will also provide more insight on the multidimensional nature of the flow structure around the baffle in a quantitative manner.

However, relatively few detailed measurements of the flow field characteristics of settling tanks with a baffle are available in the literature. Furthermore, most of them are lacking the concept that the flow has a strongly spatio-temporal nature and only qualitative description of the flow field have been made.

Considering the above reasons, the present study is mainly concerned with a comprehensive experimental investigation of the effect of a standing baffle on the vertical structure of flow and its spatio-temporal characteristics in a quantitative manner. It was realized by using ultrasonic Doppler method, by which spatio-temporal velocity fields could be obtained non-intrusively. 


\section{Experiments}

\subsection{Experimental set-up}

The experimental set up is schematically depicted in Fig. 1. It consists of an open channel, a reservoir, and a pump that circulates the tap water from the reserve tank through the return pipe and the open channel.

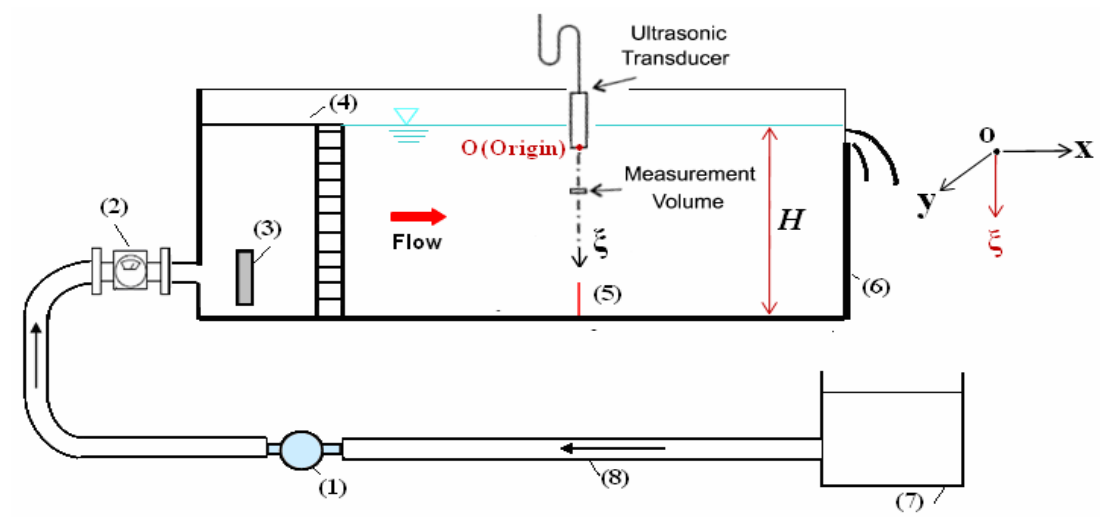

Fig. 1 Experimental apparatus representing:

(1) Pump (2) Flow meter (3) Barrier wall (4) Honey comb (5) Baffle

(6) Outlet weir (7) Reservoir (8) Return pipe

The experiments were performed in the acrylic rectangular open channel $3.5 \mathrm{~m} \times 0.3 \mathrm{~m} \times 0.3 \mathrm{~m}$ in total length, width and height, respectively. The Cartesian coordinates $x, y$ and $z$ are the longitudinal, spanwise and vertical coordinates (with $x=0$ corresponding to the baffle's position, $y=0$ corresponding to the center of channel), respectively. The downstream direction from the baffle is positive, i.e. $x>0$. $\xi(\mathrm{mm})$ is the distance from transducer's tip on the measuring line(Fig. 1). The working length of the channel is $3 \mathrm{~m}$. The first half meter was designed for conditioning of the flow.

The baffle is an acrylic rectangular plate in the middle of the channel and is extended across the full width of the channel. It was located far enough from the flow conditioner for the approaching flow to be fully developed. The height of the baffle $(S)$ is set to $60 \mathrm{~mm}$ in the current set of experiments.

Flow rate was controlled by a frequency convertor and measured by a flow meter at the inlet pipe section. The incoming flow is conditioned with a series of barrier wall and honeycombs to distribute the incoming flow all over the water layer and to reduce the background turbulence level as much as possible. The water depth $(H)$ was controlled by a sharp-edged weir at the downstream end of the channel.

\subsection{Instrumentation}

The Ultrasound Velocity Profiler (UVP) has been utilized to investigate spatio-temporal behavior of flow over a baffle. The UVP monitor used in this experiment was a UVP-Duo (Met-Flow S.A.) model. A great advantage of this method compared to the other methods such as hot-wire, ADV etc. is that it can obtain spatio-temporal information of the flow field non-invasively. Ultrasonic transducer mounted at the top of the channel (near the free surface) vertically and looking downwards. Its tip was inserted $10 \mathrm{~mm}$ below the free surface of water (Fig. 1) at the height of $h=200 \mathrm{~mm}$ above the channel bed.

The instantaneous vertical velocities along the propagation direction of the ultrasonic wave, $\xi(\mathrm{mm})$, are obtained. The basic frequency of the 
ultrasound is $4 \mathrm{MHz}$. The measurement volume has a disc shape with average diameter of $5 \mathrm{~mm}$ and thickness of $0.74 \mathrm{~mm}$.

Each instantaneous velocity profile consists of 270 measuring points that are measured simultaneously. The distance between two measurement points is $0.012 S$ and measured range is $3.32 S$. The sampling period to obtain one velocity profile was about $46 \mathrm{~ms}$ and the total measurement time for 4096 profiles was $188 \mathrm{~s}$. The water was seeded with micro tracer particles with specific density of 1.02 , and the average diameter of 60 to $200 \mu \mathrm{m}$.

\subsection{Experimental Conditions}

The geometrical dimensions provide a baffle (step) with an aspect ratio $(A R=W / S)$ of $5: 1$ and an expansion ration $(E R=H /(H-S))$ of $1.4: 1 . W$ is the channel width. Total water depth was $H=210 \mathrm{~mm}$ all over channel, achieved by setting the outlet weir of height of $180 \mathrm{~mm}$ for the selected flow rate, $Q$.

The flow conditions and Reynolds and Froude numbers defined as $R e=$ $U h / v$ and $F r=U /(g h)^{0.5}$ are shown in table 1. $v$ is kinematic viscosity of water at $T=20^{\circ} C, U=Q / A$ is the average bulk velocity, where $A$ denotes cross-sectional flow area, and $h$ is a characteristic length. Since two length scales can be considered; namely the total water depth $(H)$ and the baffle height $(S)$, two $\operatorname{Re}$ numbers can be defined as $\operatorname{Re}(H)$ and $\operatorname{Re}(S)$, respectively. The Froude number corresponds to subcritical flows. Despite its value the similitude of Froude was used, similar to Lyn and Rodi ${ }^{(18)}$ and Jamshidnia et al. ${ }^{(25)}$. In the absence of sediment transport or density differences, the Froude and Hazen numbers have no considerable effect ${ }^{(25)}$.

It is estimated that the ratio of the baffle height and the boundary layer thickness is small so the on-coming turbulent boundary layer is relatively thin.

Table 1: Flow conditions in the present experiments

\begin{tabular}{cccccc}
\hline$Q$ (lit/s) & $S(\mathrm{~m})$ & $\operatorname{Re}(H)$ & $\operatorname{Fr}(H)$ & $\operatorname{Re}(\mathrm{S})$ & $\operatorname{Fr}(\mathrm{S})$ \\
\hline 3.1 & 0.06 & 10333 & 0.034 & 2952 & 0.06 \\
\hline
\end{tabular}

\section{Results and Discussion}

A single transducer was scanned along the channel at various streamwise locations in the vertical central plane ( $x-z$ plane) of the channel. Locations of the measuring lines are presented in terms of streamwise position of the transducer tip $(x)$ from the baffle. Since the experiments were performed on the vertical central plane, 2D flow can be reasonably assumed in this plane.

Since the transducer disturbs the flow, the data near it (almost $10 \mathrm{~mm}$ from its tip) are not shown in the presented results. In the region very close to the channel bed, some part of the measurement volume is located inside the wall causing error. Thus the data of these parts are also eliminated.

\subsection{Mean Velocities and Turbulent intensities}

For investigating a mean flow field time-averaged vertical velocities are computed from a series of 4096 instantaneous velocity profiles at each measuring line. The velocity obtained by UVP is a component of the velocity vector projected on the vertical measuring line that is: on-axis velocity. Thus the on-axis velocity represents the component of the velocity on the vertical 
measuring lines hereinafter referred to as vertical velocity component. If the value of on-axis velocity becomes zero the velocity direction is perpendicular to the measuring line that is horizontal. It does not necessarily mean that the velocity has a zero value. Thus, a positive value of the on-axis (vertical) velocity means that the projection of the velocity vector on the measurement line is on the propagation direction of ultrasound $(\xi)$ that is downward.

Turbulence data are computed based on the fluctuating vertical velocity from the Reynolds decomposition. Thus, the instantaneous vertical velocity $\left(v_{\xi}\right)$ is decomposed into a mean $\left(V_{\xi}\right)$ and a fluctuation component $\left(v^{\prime} \xi\right)$ :

$$
v_{\xi}=V_{\xi}+v_{\xi}^{\prime}
$$

Profiles of the time-averaged vertical velocity with velocity fluctuations at each spatial point are illustrated in Figs. 2(a) and 3(a) for up- and downstream of the baffle, respectively. The ordinate is the distance from the transducer tip on the measuring line $(\xi)$ and the abscissa is the vertical velocity. In Figs 2 and 3 the right-hand vertical-axis represents the non-dimensional height that is $h / S$. Error bars represent the standard deviation of the vertical velocity fluctuations $\left(\sigma_{v}\right)$ at each spatial point over the measuring period. Since no smoothing has been applied to the data the uncertainties which might be related to the spatial or time resolution are small.

Investigation of the corresponding relative turbulent intensity (T.I.) profiles allows the characterization of the flow topology in a more detailed

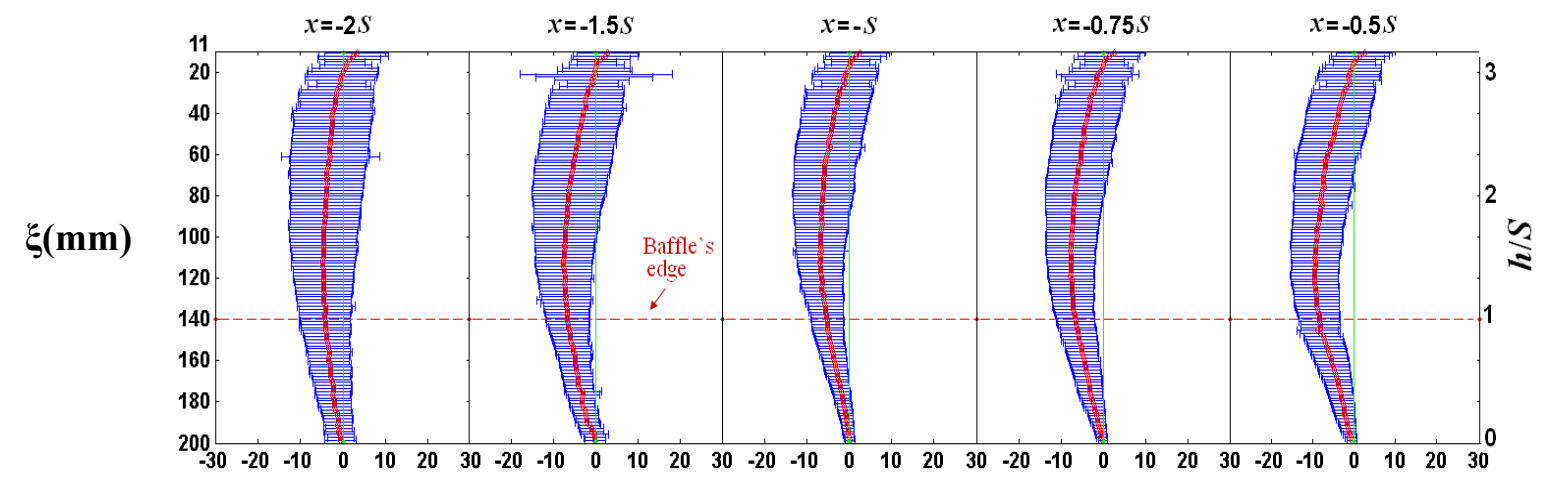

a) $V_{\xi}(\mathrm{mm} / \mathrm{s})$ at upstream sections of the baffle

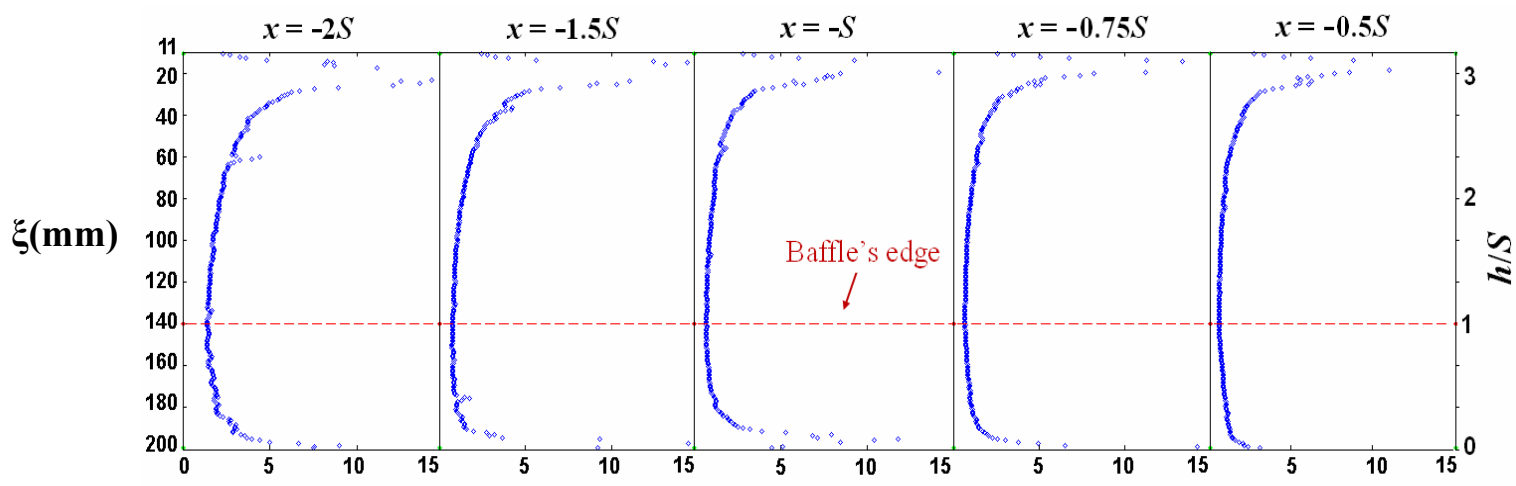

b) T.I. at upstream of the baffle

Fig. 2 (a)Time-averaged vertical velocity profiles, the error bars represent standard deviation of velocity fluctuations and (b) Profiles of relative vertical turbulent intensity component from $x / S=-2$ to $x / S=-0.5$. 
$\xi(\mathbf{m m})$

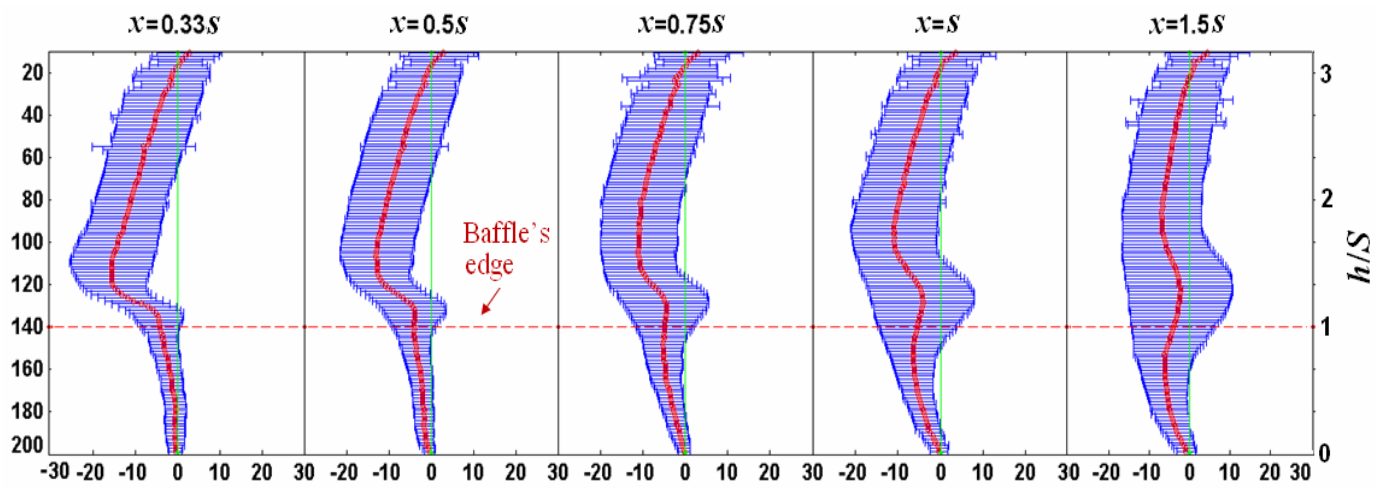

a) $V_{\xi}(\mathrm{mm} / \mathrm{s})$ at downstream sections of the baffle

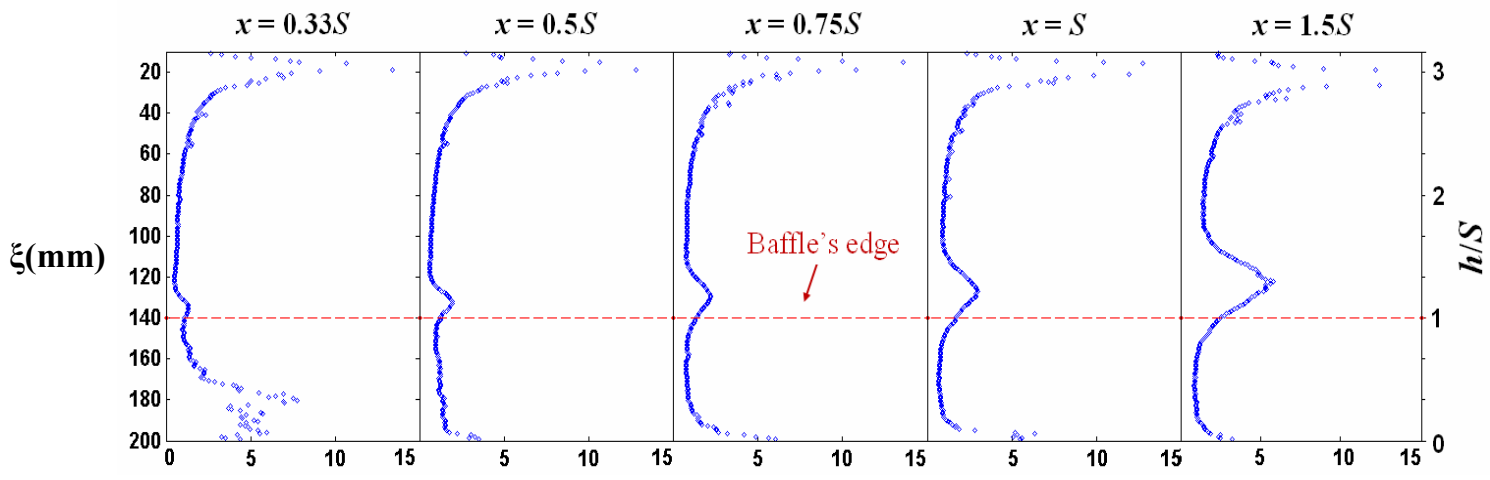

b) T.I. at downstream sections of the baffle

Fig. 3 (a)Time-averaged vertical velocity component profiles, the error bars represent standard deviation of velocity fluctuations and (b) Profiles of relative vertical turbulent intensity component from $x / S=0.33$ to $x / S=1.5$

manner. Relative turbulent intensity is defined by the following relationship:

$$
T . I .\left(\text { Relative Turbulent Intensity) }=\left|\sigma_{v} / V_{\xi}\right|\right.
$$

Figures 2(b) and 3(b) represent the profiles of relative vertical turbulent intensity components over various vertical measuring lines for up- and downstream of the baffle, respectively. The vertical axis is the distance from the transducer tip and the abscissa is the relative turbulent intensities (T.I.). It is noted that a peak in the relative turbulence intensity profiles means either a large fluctuation in velocity or a very small mean velocity.

In Figs. 2 and 3 an apparent streamwise variation in the profiles of the time-averaged vertical velocity and relative vertical turbulent intensity components is observed. The profiles of the turbulent characteristics complement the mean field profiles; e.g. non-uniformities in the mean field are reflected in the non-uniformities in the T.I. field.

Negative velocity values in the mean velocity profiles indicate existence of the uprising flow at baffle's upstream.

But at baffle's downstream (Fig.3) the flow structure is more complicated and is considerably altered as a result of the influence of the baffle. At this region, the most significant feature of the relative turbulent intensity profiles is the pronounced peak values (like a bump) near the baffle's edge. They can be mainly associated with existence of the vortex shedding behind the baffle. This effect is also reflected as local maxima (a kind of bump with a value near 
zero and a high value of relative turbulent intensity) in the mean velocities profiles at similar heights just above the baffle's edge (around $\xi=130 \mathrm{~mm}$ ) which is a representative of the vortex shedding and periodicity behind the baffle. The results also provide a detailed picture on the spatial velocity variations or the flow non-uniformity.

Behind the baffle values of the highest turbulent intensities are increasing with distance from the baffle. Also the observed bumps are spreading over the space (vertical direction). These observations can be regarded as indications of the spread of the vortex produced from the baffle edge over the space.

Furthermore, negative velocity values from the baffle edge to the channel bed are indicative of the reverse flow region at the baffle's downstream. The results also confirm the captured phenomena in our previous study ${ }^{(26)}$.

It is noted that large values of relative turbulent intensities in Figs. 2(b) and 3(b) near the free surface or near the channel bed are due to very small values of mean vertical velocities at those regions. Near the free surface and close to the transducer tip flow is almost in streamwise direction and thus its vertical component is very small. Also near the bed the flow velocity is very small.

\subsection{Spatio-temporal velocity field}

It is of value to investigate variation of velocities in space against time to capture the time dependent and unsteady nature of the flow characteristics. In Figs. $4 \mathrm{a}$ and $4 \mathrm{~b}$ typical results are shown for immediate up- and downstream of the baffle, respectively. The horizontal axis is time(s). The left-hand vertical axis is the distance $\xi(\mathrm{mm})$ of measurement points from the transducer tip and the right-hand vertical-axis represents the non-dimensional height that is $h / S$. In the figures only data values over the first 500 profiles are visualized.

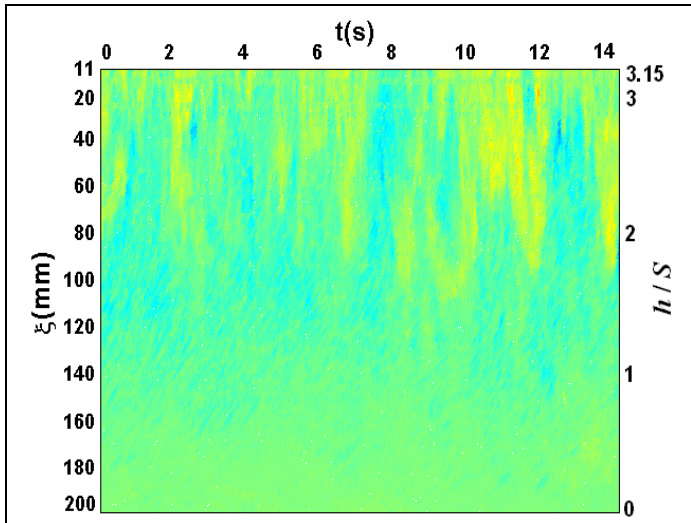

a) $v(\xi, t)$ for $x / S=-1$

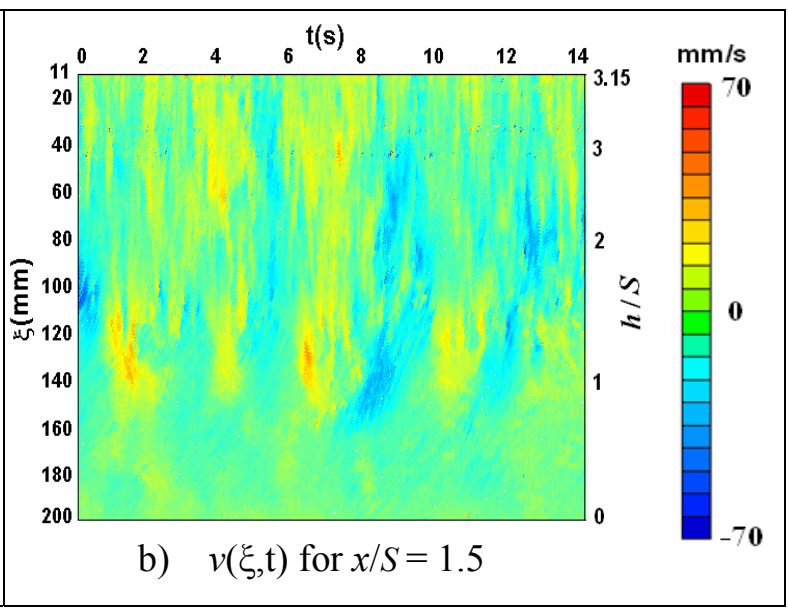

b) $v(\xi, t)$ for $x / S=1.5$

Fig. 4 Spatio-temporal velocity distribution

a) for a typical upstream sections b) for a typical downstream sections

Spatio-temporal instantaneous vertical velocity distributions, $v(\xi, t)$, at different sections indicate uprising flow at the immediate baffle's upstream as negative velocities in Fig. 4a. For baffle's downstream the existence of a periodic pattern in the flow direction near the baffle edge (around $\xi=120$ $\mathrm{mm}$ ) is confirmed. Such periodicity is not observed for upstream sections.

There are two possible oscillations behind the baffle. As behind the BFS, it is known that a large recirculation zone fluctuates and reattaching points oscillate. Another possible oscillation is by vortex shedding. The former, 
however, shows relatively slow oscillation which corresponds to a long periodicity and it might prevail in relatively large region. Here it is judged that the periodicity observed on the spatio-temporal velocity distribution would then not be by this recirculation but due to the vortex shedding at downstream of the baffle from the fact that it appears only near its edge.

On the whole, the change in the temporal structure of the flow from up- to the downstream of the baffle could be captured quantitatively in spatio-temporal distribution of vertical velocities.

\subsection{Temporal structure of the flow}

Space-dependent power spectra from vertical velocity components is extracted to understand the temporal structure of the flow on the vertical direction and to detect the temporal periodicity in the velocity. The effect of a baffle on the temporal structure is investigated in a quantitative manner.

The power spectrum obtained from raw data of velocity is usually quite noisy. To eliminate noise a smoothing procedure has been used. The $N$-point velocity time series, $u[n]$, is splitted into two $N / 2$-point time series consisting of even- and odd-numbered points in $u[n]$. Then the ensemble average of the calculated power spectra of the two subdivided time series was taken over frequency to obtain the smoothed power spectrum. Thus, the random noise could be fairly suppressed out from the resultant power spectrum.

\subsubsection{Space dependent power spectra}

Space-dependent power spectra, $P(\xi, f)$, of the vertical velocity at up- and downstream of baffle are shown in Figs. 5 and 6, respectively. The left-hand ordinate is distance, $\xi(\mathrm{mm})$, from the transducer's tip and the right-hand one is non-dimensional height, $h / S$, and the abscissa is frequency, $f(\mathrm{~Hz})$.

For upstream sections (Fig. 5) no specific peak structure is observed and the power spectra have a broad distribution whereas for downstream sections (Fig. 6) some peak structures can clearly be observed. Although there are some specific distribution of these peak structures at various downstream sections but specifically appearance of these peak structures is concentrated near the baffle edge for $f<4 \mathrm{~Hz}$. These peaks can be mainly attributed to the vortex shedding and are also reflected in the peak values of the relative turbulent intensity profiles (e.g. for $x / S=0.75$ ) and in the periodic behavior of velocity near the baffle edge at the corresponding section (Fig. 4b).

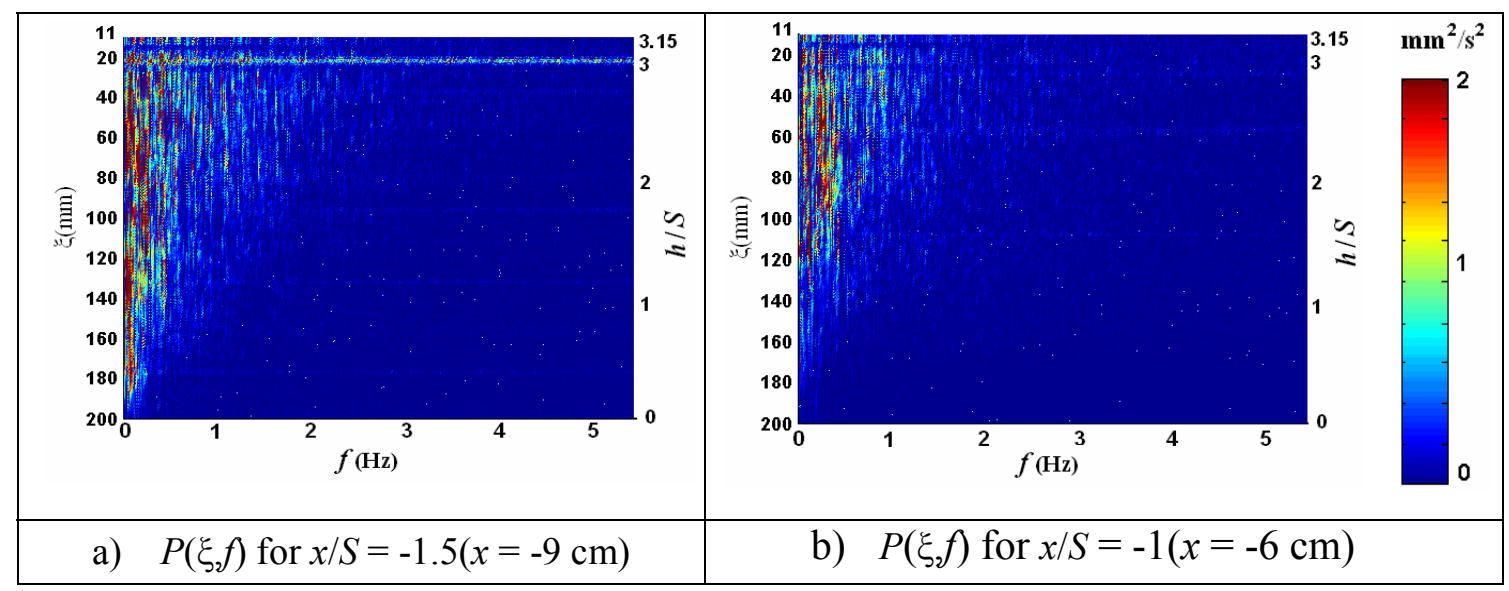

Fig. 5 Typical space-dependent power spectra of upstream sections 


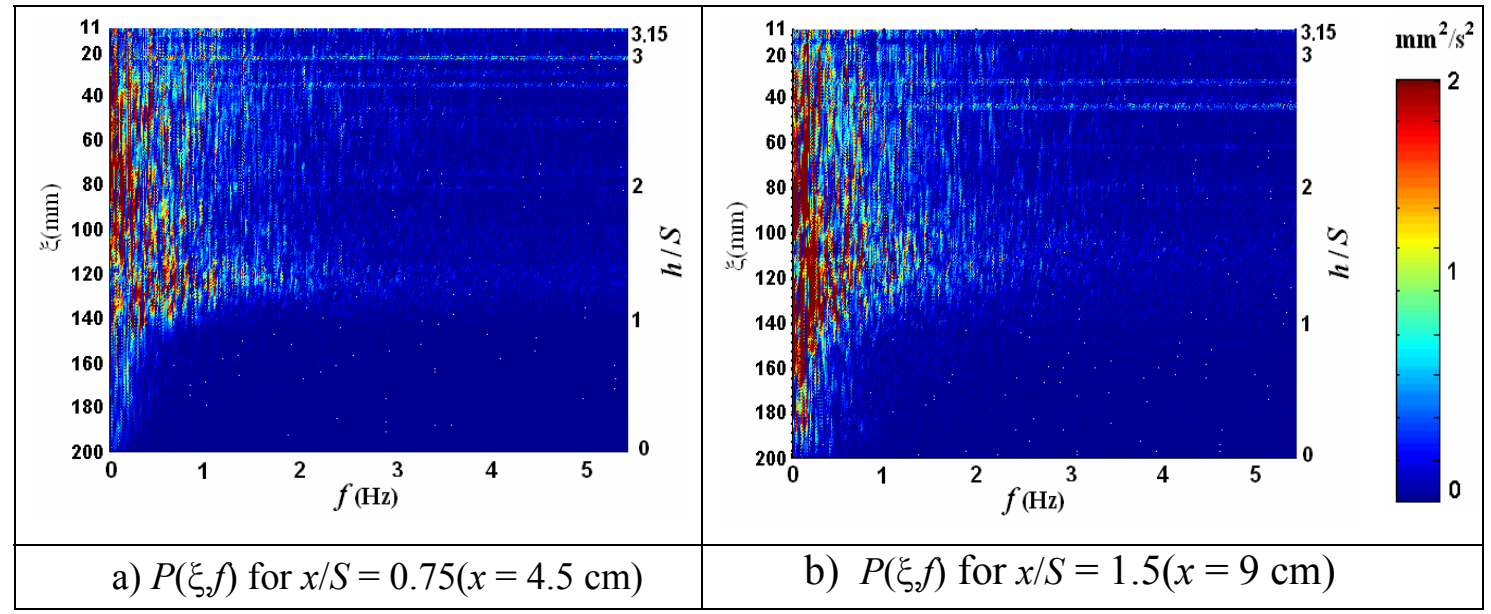

Fig. 6 Typical space-dependent power spectra of downstream sections

As a result, baffle effect in producing vortex shedding could be captured by directly measuring the power spectra of the vertical velocity component.

\subsubsection{Spatial distribution of frequency modes}

To investigate the spatial structure and periodicity of the flow in detail ensemble average of the space distribution of the power spectra over some frequency ranges (modes) $\left(f_{1}<f<f_{2}\right)$ has been calculated as follows:

$$
P_{\text {Ave. }}(\xi)=\sum_{f=f_{1}}^{f=f_{2}} P(\xi, f) / m
$$

In Eq. (3), $m$ is the number of data points in frequency domain for the corresponding frequency range. To recognize which frequency modes are of significance various frequency ranges were selected for ensemble averaging. Since the peak structures are concentrated in the $f<4 \mathrm{~Hz}$ these ranges were selected in the interval of $f<4$. By comparing space distributions of various frequency modes it was found that two frequency modes namely $\mathrm{A}(0.8 \mathrm{~Hz}<f$ $<1.2 \mathrm{~Hz})$ and $\mathrm{B}(1.5 \mathrm{~Hz}<f<2.5 \mathrm{~Hz})$ are dominant and play a major role in the appearance of peaks in the space distribution of various frequencies.

Figs. 7 and 8 show the distribution of frequency modes $\mathrm{A}$ and $\mathrm{B}$ for typical sections at up- and downstream of the baffle, respectively. The left-hand ordinate is $\xi(\mathrm{mm})$ from the transducer's tip and the right-hand one is non-dimensional height, $h / S$. The abscissa is the averaged power denoted by $P_{\text {Ave. }}\left(\mathrm{mm}^{2} / \mathrm{s}^{2}\right)$ over the corresponding frequency range.

At baffle's upstream (Fig. 7) the spatial distribution of both frequency modes $\mathrm{A}$ and $\mathrm{B}$ is uniform and no specific characteristic is observed.

Space distribution of frequency modes (A and B) in Fig. 8 at baffle's downstream indicate existence of a peak structure near the baffle edge (around $\xi=120 \mathrm{~mm}$ ). The location of such peak values is almost same as the location of the maximum values of the relative turbulent intensity profiles (e.g. at $x / S=0.75$, Fig. $3 b$ ) as well as the same periodic band in the spatio-temporal velocities distribution for those sections. Thus, the existence of vortex shedding and its consequent periodicity was reflected in the distribution of some frequency modes which was previously confirmed in the profiles of the time-averaged vertical velocity and relative turbulent intensities as well as in the spatio-temporal vertical velocity distribution. 
On the whole space distributions of the frequency modes (A and B) reveal existence of some peak values at downstream sections for the both frequency modes mainly near the proximity of baffle edge height (around $\xi=120 \mathrm{~mm}$ ) which is mainly associated with vortex shedding from baffle edge. The results are in agreement with our ${ }^{(26)}$ previous findings and confirm existence of two dominant frequency modes (A and B) for the vertical component as well.
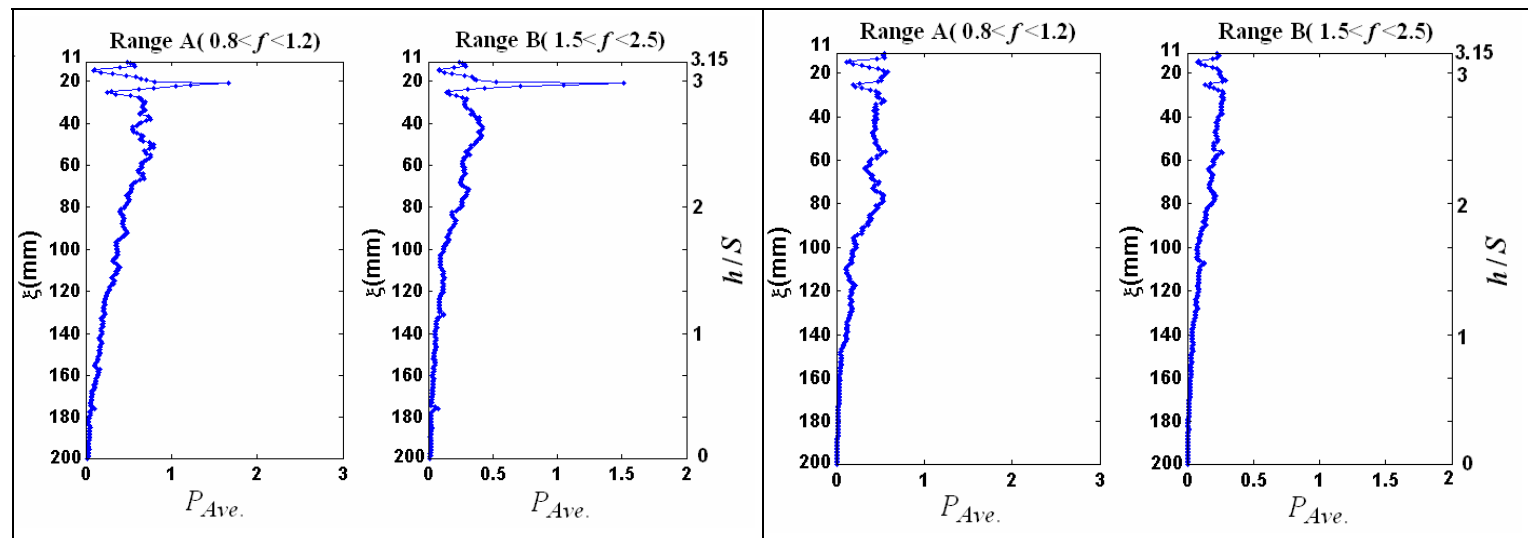

a) $x / S=-1.5(x=-9 \mathrm{~cm})$

b) $x / S=-1(x=-6 \mathrm{~cm})$

Fig. 7 Space distribution of frequency modes A and B for typical upstream sections

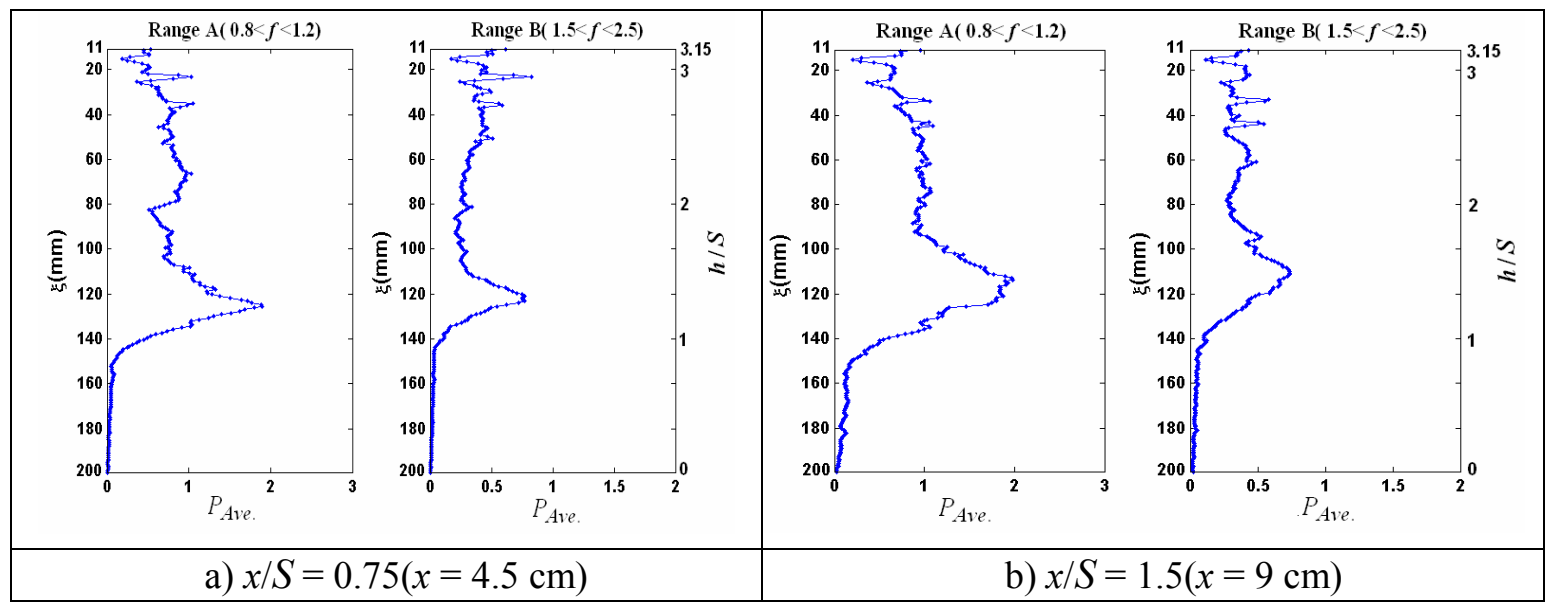

Fig. 8 Space distribution of frequency modes A and B for typical downstream sections

\subsection{Cross-correlation analysis}

The correlation coefficient between fluctuations of velocities at two points separated in the mainstream and vertical directions is useful to extract the degree of correlation between vertical fluctuating velocities at two points one at upstream and the other at downstream of the baffle to investigate the baffle's effect on the flow structure and the complex flow behavior around it comprehensively. Importantly, to the author's best knowledge there is no previous study in the literature evaluating such correlations quantitatively.

For investigation of the degree of correlation between vertical velocity fluctuations at baffle's up- and downstream points a pair of UVP transducers was used to measure the velocity components on various pairs of vertical measuring lines. One transducer is positioned at the upstream and the other at the downstream of the baffle.

It is noticeable that, such velocity information provided by UVP can not 
be obtained by pointwise measurement techniques over the space simultaneously. Also such measurements by means of other methods such as ADV (Acoustic Doppler Velocimeter) might be erroneous because the upstream probe may disturb the flow. For this reason UVP has an advantage over the other velocity measurement methods in that it can measure such quantities without possible error of probe-induced disturbance.

Figure 9 shows the position of transducers and measuring lines. Both of the transducers were positioned at the same height of $h=200 \mathrm{~mm}$ above the channel. $\xi_{\mathrm{u}}$ and $\xi_{\mathrm{d}}$ indicate the distance of measured points from transducers tip for a typical pair of up- and downstream measuring lines, respectively.

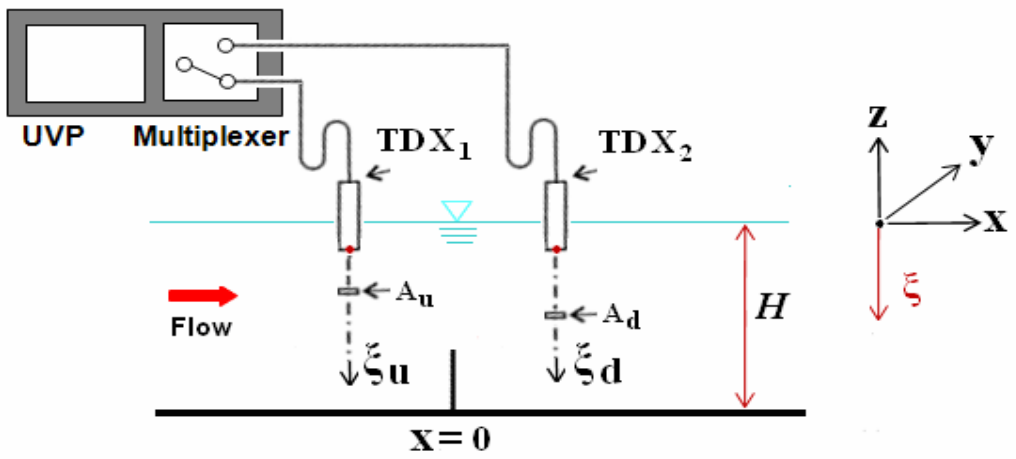

Fig. 9 Schematic of experiments using two transducers and multi-line method

The transducers, switched by a multiplexer, emitted the ultrasound beam alternatively to acquire velocity distributions along two individual measuring lines $\left(\xi_{\mathrm{u}}\right.$ and $\xi_{\mathrm{d}}$ ) almost simultaneously. For each of the two multiplexed transducers, UVP recorded vertical velocity at 270 points. The time interval to switch the transducers in the multiplexer was $\sim 0.28 \mathrm{~s}$. It is assumed that the time interval between the two consecutive velocities is short enough, and the flow velocities do not change suddenly in time or space, that is, the similarity in the flow pattern is preserved.

Measurements have been mainly performed for various pairs of up- and downstream measuring lines namely in the range of $-1 \leq x / S \leq 1.5$.

Normalized cross correlation coefficient $C_{u d}$ between the vertical velocity fluctuations at two points located at different depths one in the baffle's upstream and the other in its downstream is computed according to:

$C_{u d}(\tau)=\left(\sum_{n=1}^{N} v_{\xi u}^{\prime}[t] v_{\xi d}^{\prime}[t+\tau]\right) /\left(\sum_{n=1}^{N} v_{\xi u}^{\prime}[t]^{2} \sum_{n=1}^{N} v_{\xi d}^{\prime}[t]^{2}\right)^{0.5}$

In Eq. 4, $\tau$ is the time lag and $v_{\xi u}^{\prime}[\mathrm{t}]$ and $v_{\xi d}^{\prime}[\mathrm{t}]$ represent time series of the vertical velocity fluctuations at two points (typically shown by $A_{u}$ and $A_{d}$ in Fig. 9) one in baffle's upstream and the other at its downstream.

The maximum of the absolute value of $C_{u d}$ hereinafter referred to as "degree of correlation" for all combination of pairs of up- and downstream points located on two corresponding measuring sections namely $x / S=(-p, q)$ has been found. $p$ and $q$ represent two positive numbers.

Figure 10 shows contour maps of the degree of correlation corresponding to various typical pairs of up- and downstream measuring lines namely $x / S=(-0.75, q)$. The vertical and horizontal axes denoted by $\xi_{\mathrm{u}}$ and $\xi_{\mathrm{d}}$ 
(mm) represent distance from the transducer's tip and the $h_{\mathrm{u}} / S$ and $h_{\mathrm{d}} / S$ are non-dimensional heights for the up- and downstream measuring lines, respectively. For obtaining each color map the two point correlation coefficient has been computed $270 \times 270$ times corresponding to all combinations of up- and downstream points.

In the maps the color at each point expresses the value of degree of correlation of vertical velocity fluctuations between two points located at different depths of $\xi_{\mathrm{u}}$ and $\xi_{\mathrm{d}}$. It is noted that the main diagonal of the maps corresponds to the correlation values between points located at the same depth at up- and downstream of the baffle.

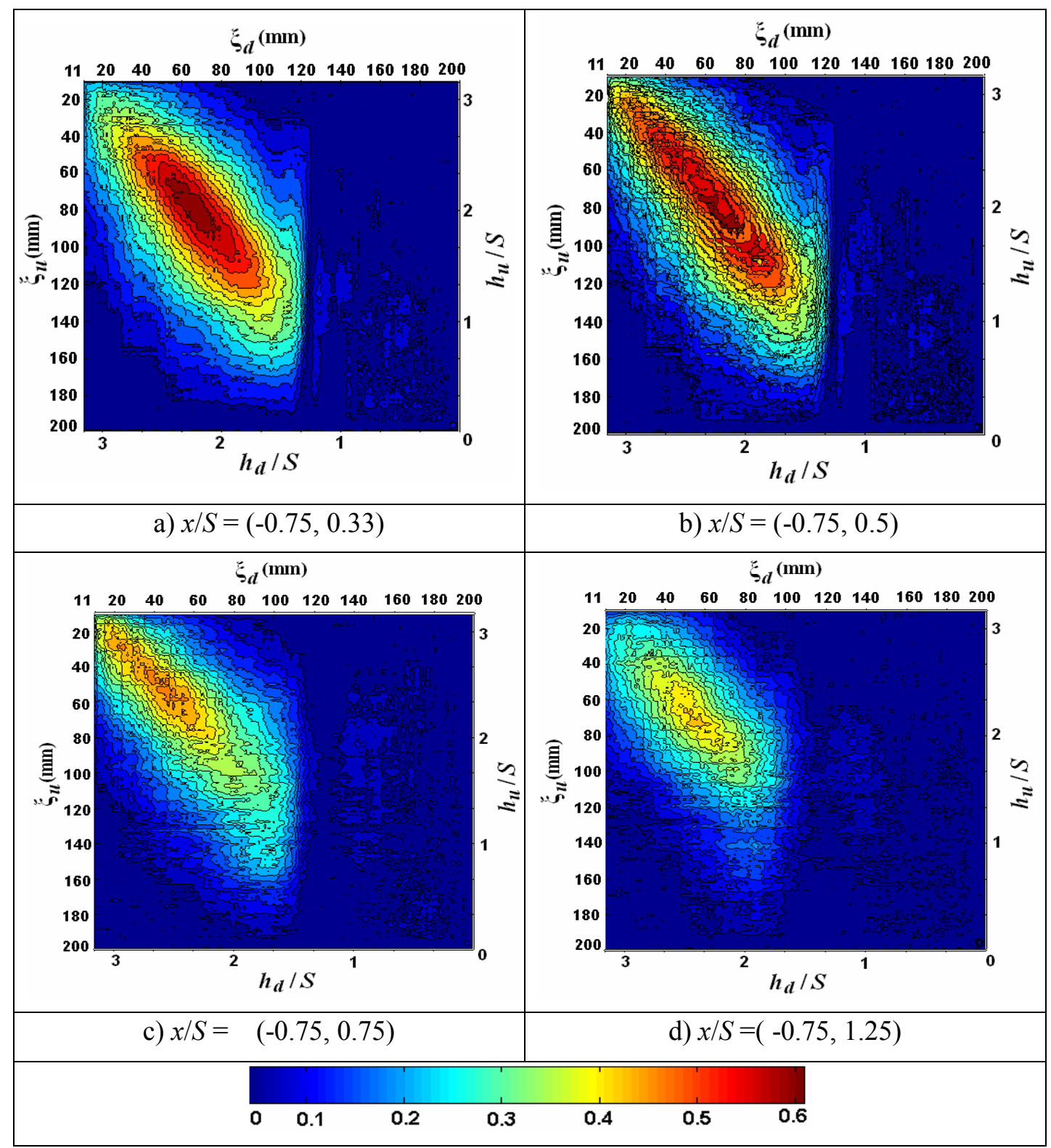

Fig.10 Typical contour maps of degree of correlation between fluctuations of vertical velocities at points upstream of baffle with that of downstream ones for various pairs of upand downstream vertical measuring lines

Figure 10 reveals the existence of two distinct regions with very different characteristics. One region with its own specific characteristics is extended from the free surface to the proximity of the baffle edge height. From Fig. 10(a) it is evident that local peak correlation values are observed 
inside this region, in a zone in between the free surface and edge of the baffle (around $\xi=80 \mathrm{~mm}$ ). It can be interpreted that this region is under the slightest influence of the baffle and free surface and thus the highest values of correlation degree exist between the corresponding velocity fluctuations inside it which indicate that turbulent velocities are transported with the flow from up- to downstream of the baffle within this region. In this region the time lag corresponding to the peak value of $C_{u d}$ in turn corresponds to the travelling time of the structure within this region from up- to downstream.

From the peak region to the free surface the values of correlation degree are gradually reduced. This can be attributed to the free surface effect on velocity fluctuations as fluid moves downstream along the free surface. It is noted that from the local peak region to the proximity of baffle edge height the value of degree of correlation is also reduces. This can be attributed to the existence of the baffle and its effect on the flow structure at its downstream.

Also in general, with increase of distance between a pair of up- and downstream measurement lines the degree of correlation between each pairs of up- and downstream points decreases. This is reasonable since one would expect a correlation coefficient between two velocity signals to decrease as the distance between their measurement locations increases. Thus, mainly high value of correlations is observed for pairs of $x / S=(-0.75, n)$ for $n=0.33$, $n=0.5$ and $n=0.75$ that are immediately behind the baffle.

Another region is extended from the channels' bed to the proximity of the edge of the baffle. In contrast to the other region no significance correlation values are observed in this zone. It can be concluded that baffle acts as a barrier wall and suddenly destroys the degree of correlation.

On the whole we could extract the characteristics of different regions around a baffle in a quantitative manner. The existence of two distinct regions with different characteristics was confirmed quantitatively.

\section{Conclusions}

A spatial and temporal vertical structure of flow over a baffle in a rectangular open channel was successfully measured by an Ultrasonic Velocity Profiler (UVP). The effect of the baffle on this flow structure has also been discussed. Based on the results the following conclusions can be drawn:

1. The mean flow analysis shows how the flow structure changes from up- to downstream of the baffle. For the upstream of the baffle uprising flow and for its downstream vortex shedding, reverse flow region were captured by paying attention to the distribution of the mean vertical velocities as well as peak values of the relative turbulent intensity profiles.

2. Behind the baffle development of time dependent flow due to the vortex shedding was clearly observed as periodicity in the spatio-temporal distributions of vertical velocities.

3. Space-dependent power spectra of vertical velocities reveal the existence of some peak structures over space at downstream sections of the baffle whereas at its upstream such peak structures are not observed. Also existence of dominant peak values mainly in the space distribution of two frequency modes was observed at baffle's downstream. The existence of peak structures in the space-dependent power spectra and peak values in two frequency modes downstream of the baffle are in agreement with the appearance of the peak values in the relative turbulence intensity profiles 
and the periodic behavior of spatio-temporal velocity distribution for the corresponding sections near baffle edge height. Thus such peak structures can be attributed to the vortex shedding due to existence of the baffle.

4. Importantly, by multi-line method, color maps of the peak of the absolute value of the normalized cross-correlation coefficients between vertical velocity fluctuations at upstream points with that of downstream ones could be obtained. The results were used to evaluate the effect of the baffle on their degree of correlation. Also, existence of two regions with various characteristics has been confirmed. In one region extended from the proximity of the baffle edge height to channel bed the degree of correlation between vertical velocity fluctuations at baffle's up- and downstream points is strongly damped out. Furthermore, the existence of a peak region inside the other region which is extended from the free surface to baffle's edge has been confirmed. In this zone the degree of correlation decreases gradually from the peak region to the proximity of the baffle edge height which is indicative of baffle effect on the flow structure in its downstream. Also the decrease in the correlation degree from the peak to the free surface was captured which may be attributed to the effect of free surface. As a future step authors plan to conduct vector measurements by UVP to capture the flow field in a more detailed way.

\section{Acknowledgments}

First author is grateful to Ministry of Education and Science (MEXT) of Japan for providing the Japanese Government Scholarship for the PhD study.

\section{References}

[1] Barkley D., Gomes M. G. M. and Henderson R. D., (2002), "Three-dimensional instability in flow over a backward-facing step", Journal of Fluid Mechanics, Vol. 473, pp.167-190.

[2] Chen Y.T., Nie J.H., Hsieh H.T. and Sun L.J., (2006), "Three-dimensional convection flow adjacent to inclined backward-facing step", International Journal of Heat and Mass Transfer, Vol. 49, pp.4795-4803.

[3] Lin C.W., (2006), "Experimental study of thermal behaviors in a rectangular channel with baffle of pores", International Communications in Heat and Mass Transfer, Vol. 33, Issue 8, pp. 985-992.

[4] Furuichi N., Takeda Y. and Kumada M., (2003), "Spatial structure of the flow through an axisymmetric sudden expansion", Experiments in Fluids, Vol. 34, No. 5, pp. 643-650.

[5] Armaly B.F., Durst F., Pereira J.C.F. and Schönung B., (1983), "Experimental and theoretical investigation of backward-facing step flow", Journal of Fluid Mechanics, Vol. 127, pp.473-496.

[6] Toshitake A. and Shakouchi T., (2004), "Flow characteristics over forward facing step and through abrupt contraction pipe and drag reduction", Res. Rep. Fac. Eng. Mie Univ., Vol. 29, pp. 1-8.

[7] Papadopoulos G. and Otugen M.V., (1995), "Seperating and reattaching flow structure in a suddenly expanding rectangular duct", Journal of Fluids Engineering, Vol. 117, pp.17-23.

[8] Aloui F. and Souhar M., (2000), "Experimental study of turbulent asymmetric flow in a flat duct symmetric sudden expansion", Journal of Fluids Engineering, Vol. 122, pp.174-188.

[9] Largeau J.F. and Moriniere V., (2007), "Wall pressure fluctuations and topology in 
separated flows over a forward-facing step", Experiments in Fluids, Vol. 42, pp. 21-40. [10] Tachie M.F., Balachandar R. and Bergstrom D.J., (2001), "Open channel boundry layer relaxation behind a forward facing step at low Reynolds numbers", Journal of Fluids Engineering, Vol. 123, pp. 539-544.

[11] Aloisio G., Camussi R., Ciarravano A., Di Feilice F., Di Marco A., Felli M., Fiorentini E., and Pereira F, (2007), "Velocity and wall pressure correlations over a forward facing step", Advances in Turbulence XI, Vol. 117, Proceedings of the $11^{\text {th }}$ Euromech European Turbulance Conference, Portugal.

[12] Camussi R., Felli M., Pereira F., Aloisio G. and Di Marco A., (2008), "Statistical properties of wall pressure fluctuations over a forward-facing step", Physics of Fluids, Vol. 20, pp. 1-13.

[13] Hager, W.H., Bretz, N.V., (1986), "Hydraulic jumps at positive and negative step", Journal of Hydraulic Research, Vol. 24, No.4, pp. 237-253.

[14] Hager, W.H., Sinniger, R., (1985), "Flow characteristics of the hydraulic jump in a stilling basin with an abrupt bottom rise", Journal of Hydraulic Research, Vol.23, No.2, pp. 861-866.

[15] Campbell B.K. and Empie H.J., (2006), "Improving fluid flow in clarifiers using a highly porous media", Journal of Environmental Engineering, Vol. 132, No. 10, pp. 1249-1254.

[16] Ahmed F.H., Kamel A. and Abdeljavad S., (1996), "Experimental determination of the optimal location and contraction of sedimentation tank baffles", Water, Air and Soil Pollution, Vol.92, No. 3-4, pp. 251-271.

[17] Shiono, K. And Teixeira, E.C., (2000), "Turbulent characteristics in a baffled contact tank", Journal of Hydraulic Research, Vol.38, No.4, pp. 271-278.

[18] Lyn A. and Rodi W., (1990), "Turbulence measurements in model settling tank", Journal of Hydraulic Engineering, Vol.116, No. 1, pp. 3-21.

[19] Bretscher U., Krebs P. and Hager W.H., (1992), "Improvement of flow in final settling tanks", Journal of Environmental Engineering, Vol. 118, No. 3, pp. 307-321.

[20] Taebi Harandi A. and Schroeder E.D., (1995), "Analysis of structural features on performance of secondary clarifiers", Journal of Environmental Engineering, Vol. 121, No. 12, pp. 911-919.

[21] Taebi Harandi A. and Schroeder E.D., (2000), "Formation of density currents in secondary clarifiers", Journal of Water Research, Vol. 34, No. 4, pp. 1225-1232.

[22] Tamayol A., Firoozabadi B. and Ahmadi G., (2008), "Determination of settling tanks performance using an Eulerian-Lagrangian method", Journal of Applied Fluid Mechanics, Vol. 1, No. 1, pp. 43-54.

[23] Firoozabadi B., Jamshidnia H.R. and Moslemi M., (2007), "Experimental investigation of the effect of baffle on the flow pattern in primary settling tanks", The $15^{\text {th }}$ Annual International Mechanical Engineering Conference, Tehran, Iran.

[24] Jamshidnia H.R., (2007), "Study of flow pattern in rectangular sedimentation channels." MS thesis, Sharif University of Technology, Tehran, Iran.

[25] Jamshidnia H.R., Takeda Y. and Firoozabadi B., (2010), "Effect of a standing baffle on the flow structure in a rectangular open channel", Journal of Hydraulic Research, Vol. 48, No. 3, pp. 400-404.

[26] Jamshidnia H.R. and Takeda Y., (2009), "UVP measurement of flow around a baffle in a rectangular open channel", Journal of Fluid Science and Technology, Vol. 4, No. 3, pp.758-774.

[27] McCaffrey W.D., Chouxa C.M., Baasa J.H. and Haughton P.D.W., (2003), "Spatio-temporal evolution of velocity structure, concentration and grain size stratification within experimental particulate gravity currents", Journal of Marine and Petroleum Geology,Vol.20, pp. 851-860. 